\title{
Gestión de la motivación en escenarios organizacionales
}

\section{Motivation management in organizational scenarios}

\author{
Alejandro Leon Contreras Giraldo ${ }^{1}$
}

Palabras

Claves:

Organización, gestión, líder escenarios, motivación resultados.

\begin{abstract}
Objective: this article exposes the relationship between the motivation of employees and the achievement of results within an organization, highlighting the importance of the leader in the framework of the intrinsic and extrinsic nature of motivation.

Methodology: study the role of satisfaction of needs in motivation according to the theory of selfdetermination, and how, in the opposite way, management can discourage performance.

Results and conclusions: a documentary review was carried out under the focus of qualitative research, focused on the management of the will as a focus of investment in the various scenarios for organizations and as a critical factor in the results.
\end{abstract}

Keywords:

Organization

Management Leader,

Scenarios,

Motivation

Results. \begin{abstract}
el desempeño.
Resultados y conclusiones: se realizó una revisión documental bajo el enfoque de la investigación cualitativa, centrada en la gestión de la voluntad como foco de inversión en los diversos escenarios para las organizaciones y como factor crítico en los resultados.

Resumen

Objetivo: este artículo expone la relación entre la motivación de los colaboradores y el logro de los resultados al interior de una organización, resaltando la importancia del líder en el marco de la naturaleza intrínseca y extrínseca de la motivación.

Metodología: estudiar el papel que cumple la satisfacción de necesidades en la motivación según la teoría de la autodeterminación, y cómo, de manera opuesta, la gestión gerencial puede desestimular
\end{abstract}

1Universidad Autónoma del Caribe. 


\section{Introducción}

"No es sabio el que sabe dónde está el tesoro, sino el que trabaja y lo saca"

Francisco de Quevedo (1580-1645)

La motivación, del latín movere, que traduce al verbo mover, corresponde en el escenario organizacional al esfuerzo que hace una persona para aportar valor al negocio. La calidad de esta energía tiene importantes determinantes: en su cantidad, determina la fuerza que ejerce sobre la labor; en su foco, la baja dispersión aumenta el efecto sobre los resultados, y en la duración, constituye la constancia que en definitiva supera y vence las dificultades [1].

La gestión de la voluntad es transversal al éxito en todas las organizaciones, ya que habita en el tránsito hacia los resultados en términos de productividad, y requiere en sí misma la intervención, por acción u omisión, del líder que la pretende.

La motivación es un estado deseable tanto para el individuo como para un grupo, e incluso existen varios sistemas motivacionales de naturaleza aversiva: como el dolor, el hambre, la angustia y el castigo [2]. Esta energía se desarrolla desde dos polos que convergen, uno de adentro hacia afuera: la intrínseca, y el otro desde afuera hacia adentro: la extrínseca.

El primero corresponde a la afinidad por el sentido de la acción. Es el estímulo interno de realizar un esfuerzo, la acción mediada por el deseo autónomo, consciente o inconsciente de iniciar, mantener y culminar una actividad. El segundo, por el lugar de donde proviene, desde afuera, es la acción provocada por otras personas o por el ambiente, externa, en resumen, al individuo.

La motivación extrínseca es la más común y corticalmente utilizada en los escenarios organizacionales. Tradicionalmente, para motivar a los colaboradores, se echa mano de: bonos, obsequios, premios, reconocimientos públicos o adjudicación de protagonismo; lo cual es aplicado por el empleador o jefe de proceso. La motivación intrínseca viene con el candidato que inicia un empleo y se mantiene, se refuerza o se destruye conforme evoluciona en el trasegar de la labor y su relación con el entorno [2].

En este artículo se expone la relación existente entre la motivación de los colaboradores y el logro de los resultados al interior de una organización. En este contexto, se resalta la importancia del líder en el proceso motivacional de los grupos de personas. Para todo ello, se realizó una investigación cualitativa de tipo documental, en la que se revisaron 17 fuentes comprendidas en artículos, tesis y capítulos de libros encontradas a través de buscadores disponibles en el Internet. 


\section{Revisión de la literatura}

\section{Hallazgos}

\section{La Autodeterminación}

Esta teoría se basa en tres necesidades psicológicas existentes en los seres humanos, cuya satisfacción determina la motivación de una persona. La primera es la autonomía, que supone la capacidad por parte del individuo de decidir con voluntad propia para ser autor activo de la propia historia. En este sentido, las implicaciones de las decisiones propias con respecto al entorno, generan un compromiso con el sentido de la actividad que se manifiesta en acciones como expresarse abiertamente, darle importancia a los deseos y perseguir sus propios anhelos [3, 4, 5].

La segunda es la competencia, que se refiere a la capacidad de hacer, sentir, resistir la presión, afrontar los retos, proponer ideas y superar obstáculos. No tiene que ver con el sentido de la competición, sino con la confianza en sí mismo y con el efecto que se logra al sentirse competente.

La tercera es el vínculo, que se conforma con el sentido de valoración desde y hacia el otro, el afecto, la pertenencia a grupos el relacionamiento interpersonal y la presencia en el mapa social de la realidad que habita $[6,7,8,9]$.

Así, según el nivel de satisfacción de estas necesidades se activa la motivación intrínseca o la extrínseca. Por ejemplo, si la persona tiene una idea en su trabajo, la propone, la defiende y la ejecuta, mantendrá durante el proceso una motivación intrínseca pues sentirá que tiene un nivel de control sobre su pensamiento y su creatividad, sobre lo que expresa y defiende. Incluso, al recibir el rol social dentro del grupo en el que argumenta, recibirá estimulación sobre la necesidad del vínculo.

Pero si la idea es impuesta de manera no concertada por parte del jefe, limitando la posibilidad de opinión, el empleado se sentirá en un rol social irrelevante, en el que su capacidad no se expresa, y ellos dará como resultado una desmotivación por insatisfacción de la autonomía, la capacidad y el vínculo. En el primer caso, la energía motivacional que se activa es la intrínseca; en el segundo caso, un estímulo aversivo de naturaleza extrínseca limita la motivación. Ahora bien, si se tratara de un estímulo aversivo relevante para el trabajador, podría ser suficiente para activar una respuesta de defensa, que en sí misma, ya con un foco diferente, ese convierte en energía motivacional intrínseca pura, negativa para los intereses de cualquier organización. 


\section{Antecedentes conductistas}

A pesar de que la importancia de la motivación es totalmente reconocida, los líderes suelen desconocer la profundidad del tema, y en la mayoría de las situaciones usan técnicas conductistas que se conocen como "zanahoria y garrote" [10].

Estas técnicas se enfocan en recompensas tangibles o en castigos. Pero, si bien la "zanahoria y el garrote" puede resultar en un aumento de productividad a corto plazo, lo más probable es que, a largo plazo, genere desmotivación e incluso fomente el fraude y el engaño. Este efecto negativo puede darse porque los empleados sienten que se les debe "pagar" por participar en una actividad $y$, en consecuencia, buscar los medios para lograr esas recompensas, así estos no sean transparentes.

El "garrote", por otro lado, puede generar comportamientos defensivos, paranoicos o incluso de retaliación. Cuando un empleado se siente amenazado, atemorizado por la posible pérdida de su trabajo o por cualquier otro tipo de castigo, con frecuencia puede mostrarse indiferente, poco comprometido, desmotivado o desleal [11].

Hoy en día se sabe que la motivación se relaciona en forma directamente proporcional con la contribución de valor al negocio a través del desempeño, y que este requiere una gestión efectiva del líder que interviene en un equipo de colaboradores.

La gestión del capital humano es el eje estructural de la generación de valor al negocio, pues trabaja en un activo intangible de alto costo y responsable de la viabilidad de una inversión: la creatividad y el compromiso de las personas que conforman el equipo de trabajo. Por eso dicha gestión constituye una responsabilidad estratégica de los líderes respecto a los equipos, y concretamente la de entender y profundizar en el conocimiento de las personas y sus respuestas ante estímulos [12].

\section{La motivación, ¿un intangible gestionable?}

La respuesta de esta pregunta se relaciona con la complejidad de los temas inherentes al interior de las personas. En este sentido, tanto la motivación intrínseca como la extrínseca hacen referencia a la intimidad del individuo, es decir, todas las motivaciones son internas y obedecen a la lógica natural del libre albedrío, por lo que son también de una baja predictibilidad. Debido a lo anterior, los modelos teóricos sobre la motivación ocasionan en la práctica respuestas variables y sobre los equipos de personas.

No obstante, en ausencia de recetas que aseguren respuestas predecibles, existen modelos teóricos basados en la evidencia, que ayudan a tener un criterio significativo sobre las decisiones en torno a la gestión del capital humano [1].

Evidentemente, el ser humano siempre actúa por alguna razón. El problema fundamental es que esa razón está más allá de la actuación observable, tiene un componente espiritual del que el mismo ser humano puede no ser consiente. A pesar de todo, ese componente interior no es, por ello, menos real [13].

En realidad, la relación o relacionamiento tiene un aspecto propio, singular y profundo, que no proviene de la interacción con ningún entorno. Este es determinado por necesidades que deben 
satisfacerse, y posee un aporte externo en el que aparecen conductas orientadas a horizontes definidos por el exterior, necesidades adaptativas del organismo y la coordinación social, que en gran medida son determinadas, a su vez, por los valores dominantes en una cultura, que luego son internalizados por los individuos y orientan su conducta.

Así, la conducta es causada por la cultura y por los procesos psicológicos y fisiológicos del individuo [14]. Se trata de una manifestación de sus motivaciones, que, por acción u omisión, conlleva a distintas intervenciones de la realidad. El ser humano no actúa en el vacío, sino en la sociedad, y en ella su actuación es influenciada por otras personas, mientras el influencia, a su vez, la actuación de otros [13].

\section{Gestión gerencial}

La motivación de un equipo de colaboradores requiere una intervención gerencial, pues la posición privilegiada de su rol lo hace estratégicamente visible y, por ende, influenciador del colectivo, donde encontrará la referencia que le dará sentido al hacer y al sentir en el trabajo.

Ahora bien si, como va quedando claro, son los empleados quienes hacen la ventaja competitiva, entonces es necesario diseñar un proceso de medición y gestión.

En el entorno empresarial existen prácticas de este tipo con las que se hacen mediciones sobre el impacto financiero del capital humano, pero es poco común que sean el foco de la gestión. En cambio, es frecuente encontrar a gerentes que saben en detalle el costo de las personas que dirigen, mas no suele ocurrir que sepan cuanto aportan a sus resultados en términos financieros, algo así como el estado de resultados de cada empleado.

En el renglón de los costos, esta visión de las personas es corta, pues las personas son activos, y en la medida que apalancan los ingresos y la contribución neta, constituyen un valor para la compañía.

Básicamente. una posible consecuencia de esta miopía estratégica es que se gestione como costos a un activo productivo y, por ende, se controle a la baja, con la base de que a menores costos, el aparato productivo refuerza sus eficiencias. Asumir a las personas como valiosos activos, permite llevar a cabo una gestión ubicada en el contexto de la llamada generación integral de valor [15].

Desde este punto de vista, la motivación del equipo de trabajo requiere inversiones de capital y para que este sea liberado con ese fin, debe demostrarse el retorno para los inversionistas en términos objetivos. Por otra parte, la medición sobre el particular tiene en sí misma un impacto en los colaboradores del líder, quienes se convierten de este modo en una representación cuantificada del desempeño según el esfuerzo realizado por ellos mismos.

Es así como la motivación resulta ser el elemento fundamental para los resultados, por lo que es necesario hacer inversiones financieras en su mejoramiento y, como en toda inversión, también es necesario cuantificar su retorno [15].

Corresponde contextualizar en este escenario las mediciones del impacto económico de la gestión del capital humano como una útil herramienta gerencia en la toma de decisiones. Y, a partir de esta, 
se deben definir las estrategias de motivación del equipo, cuantificar la inversión que requieren, el margen que se debe esperar y la forma de ser medidas.

En todos los casos es fundamental cuantificar la inversión en cada persona, es decir, el costo fijo/ variable de la inversión, con una representación de estos en los costos del producto que genera el ingreso financiero para que el ejercicio se complete.

Un adecuado equilibrio entre inversión, motivación, productividad y medición complementa una estrategia alineada con el mejoramiento y el éxito, lo que redunda en generación de valor a los inversionistas, proveedores, empleados y clientes.

\section{Sistemas de incentivos}

Los planes de incentivos surgen como estrategias motivacionales para promover buenos resultados dentro de las compañías, con intervenciones de premio o castigo que se construyen con fórmulas relacionadas de manera directa con la acción deseada [7].

Según la teoría de Herzberg, esas condiciones diseñadas y administradas por la empresa se encuentran por fuera del margen de control de los empleados, constituyéndose en "factores higiénicos" de naturaleza extrínseca del mismo tipo que el salario, los beneficios sociales, el tipo de dirección o supervisión, las condiciones ambientales de trabajo, el estatus, las bonificaciones, entre otros [16].

Estos factores generan un contexto de entregables adicionales a cambio del trabajo, y constituyen una mecánica psicológica en la que la preocupación por la actividad en sí se remplaza por la remuneración adicional ofrecida. De este modo se evita la insatisfacción de los empleados, pero en sí mismas no generan una satisfacción consistente, pues esta no es sostenible en el tiempo y no se enfoca hacia la motivación interna, intrínseca por la labor. En esta medida es común encontrar que los planes de incentivos son paradójicamente "planes de insatisfacción".

Por esta razón se propone realizar estrategias motivacionales basadas en la tarea, es decir, enfocadas en el reto, en las condiciones laborales dispuestas para la realización eficiente de la tarea, y que no se hallen a disposición del proceso. Se requiere reforzar la creatividad, el crecimiento personal, la inserción social satisfactoria, acompañamiento en el aprendizaje, con dosis frescas y constantes de exigencia, que permitan el error, el mejoramiento y generen la expectativa de oportunidad [17, 18].

La satisfacción de estas necesidades es infinita y, por tanto, es también una fuente de motivación constante, disponible para los líderes capacitados y dispuestos a generar sistemas colaborativos de crecimiento mutuo $[17,19]$. 


\section{Conclusión}

La motivación es un estado individual de la voluntad de las personas, que determina su disposición para realizar cualquier esfuerzo. Es una movilización de energía que afecta el entorno, y enriquece cualquier escenario organizacional. Con otras palabras, la motivación es la estructura que sostiene los resultados, pero por su naturaleza humana, variable e impredecible, es preciso que exista un líder sensible y capacitado, que entienda la importancia del capital humano y concretamente el papel de la misma en el proceso eficaz de producción.

Las organizaciones de hoy entienden que de sus colaboradores son afectados por el tipo de motivación que presentan. Por esto, la gerencia moderna la gestiona como un eje fundamental del activo humano, que requiere inversiones de capital y esfuerzo y que, como cualquier inversión que produce un retorno real, puede ser medido.

Toda persona que se encuentre en una posición de liderazgo debe entender su importancia para el equipo de personas que preside y fomentar entre sus propias capacidades la gestión de la voluntad de manera responsable. 


\section{Referencias}

1. C. Sanchez, "Motivación, satisfacción y vinculación. ¿Es gestionable?", Accion Psicologica, pp. 9-28, 2008.

2. M. M. Soriano, "La Motivacion, pilar basico de todo tipo de esfuerzo", Proyecto Social: Revista de Relaciones Laborales, pp. 163-184, 2001.

3. L. Gonzalez, I. Castillo Fernández, M. L. García Merita, \& I. Balaguer Solá, “Apoyo a la autonomía, satisfacción de las necesidades psicológicas y bienestar: Invarianza de un modelo estructural en futbolistas y bailarines", Revista psicologica del deporte, pp. 121-129, 2015.

4. M.Giusti, "Autonomía y reconocimiento. Ideas y valores", Revista Colombiana deFilosofía, pp.39-56, 2007.

5. J. Falk, Los fundamentos de una verdadera autonomía. Infancia: educar de 0 a 6 años, pp. 22-31, 2009.

6. E. Barberá Heredia, “Marco conceptual e investigación de la motivación humana”, REME, 1999. 7. V. Bedodo Espinosa, \& C. Giglio Gallardo, Motivacion Laboral y Comensaciones: Una investigacion de orientacion teórica. Santiago, Chile: Universidad de Chile, 2006.

8. M. S. Granovetter, “La tuerza de los vínculos débiles”, Politica y Sociedad, pp. 41-56, 2000.

9. J. A. Vargas Tellez, "Implicaciones de la Teoria Motivacional de la Autodeterminacion en el ámbito laboral", Revista Electronica Nova Scientia, pp. 154-175, 2007.

10. J. B. Fuentes Ortega, \& E. Q. Romero, “Los dos principios irrenunciables del análisis funcional de la conducta y del conductismo radical”, Psicothema, pp. 555-562, 2004.

11. A. Muñoz Restrepo, \& M. Ramírez Valencia, La motivacion de los empleados: Mas alla de "la zanahoria y el garrote". Enhancing student motivation through autonomysupportive AD-Minister, pp. 143 - 160, 2014.

12. R. M. Ryan, \& E. L. Deci, (2000). “La Teoría de la Autodeterminación y la Facilitación de la Motivación Intrínseca, el Desarrollo Social, y el Bienestar”, American Psychological Association, pp. 68-78, 2000.

13. A. T. Garcia Parra, “Una nueva teoria de motivacion: El modelo Antropologico de Juan Antonio Perez Lopez", Revista Puertoriqueña de Psicologia, pp. 123-163, 2004.

14.S. D'Anello Koch, “Motivación de logro, actitud hacia el trabajo, satisfacción laboral y compromiso organizacional”, Revista Interamericana Psicologia Ocupacional, pp. 21-34, 1994. 
15. J. L Torres Ordoñez, “Enfoques para la medicion de la gestion del capital humano en los resultados del negocio", Pensamiento y Gestion, pp. 151-176, 2005.

16. J. F. Manso Pinto, El legado de Frederick Irving Herzberg. Universidad EAFIT, pp. 80-86, 2002. 17. L. W. Rue, Administracion: Teoria y aplicaciones. Colombia: Alfaomega, 2000.

18. L. Ferrer Castellanos, K. González Insignares and L. Mendoza Vega, "La innovación como factor clave para mejorar la competitividad de las pymes en el departamento del Atlántico, Colombia", Dictamen Libre, no. 16, pp. 21-36, 2015.

19.M. Parra and S. Dúran, “Desarrollo organizacional y estrategias de tecnoformación en instituciones de educación superior colombianas”, Dictamen Libre, no. 1415, pp. 14-23, 2014. 